\title{
Evaluation of a shortened cardiac MRI protocol for left ventricular examinations: diagnostic performance of T1-mapping and myocardial function analysis
}

Jonathan Nadjiri ${ }^{* *} \mathbb{D}$, Anna-Lena Zaschka', Alexandra S. Straeter ${ }^{1}$, Andreas Sauter ${ }^{1}$, Maximilian Englmaier ${ }^{1}$, Florian Weis ${ }^{2}$, Karl-Ludwig Laugwitz ${ }^{2}$, Ernst J. Rummeny ${ }^{1}$, Daniela Pfeiffer ${ }^{1}$ and Michael Rasper ${ }^{1}$

\begin{abstract}
Background: In this study we sought to retrospectively evaluate whether a very brief cardiac magnetic resonance imaging (CMR) protocol sufficiently distinguishes patients with relevant myocardial changes with need for further examination from healthy subjects.

Methods: Patients with clinical indication for CMR $(n=160)$ were included in the study. Patients were categorized into two groups depending on presence of left ventricular (LV) dysfunction. ROC-analysis was done for results of T1-, T2- mapping and extracellular volume evaluation in patients without LV dysfunction. Binary endpoint was correctly depicted pathology of the conventional qualitative CMR techniques and report.

Results: In the patient cohort without LV dysfunction (49\%), AUC for T1 mapping was 82\% ( $p<0.001)$, 60\% for T2 mapping $(p=0.1)$ and $79 \%$ for ECV $(p<0.001)$. T1 mapping was significantly superior to T2 mapping to rule out left ventricular pathology $(p=0.012)$. Sensitivity for the combined use of T1 mapping and sBTFE cine imaging was 98\%; the negative predictive value was 90\%. In 49 patients (30\%) full protocol CMR did not provide any additional information; T1 mapping correctly detected $57 \%$ of the subjects from this group who would not benefit from additional CMR.

Conclusion: A shortened CMR protocol comprising T1 mapping and LV-function analysis seems suitable to rule out myocardial alterations. Every third patient of the study population did not benefit from full contrast enhanced CMR. The shortened protocol correctly identified every fifth patient who would not benefit but no relevant pathologic findings with the obligation for treatment were missed.
\end{abstract}

Keywords: Cardiac MRI, Economic, Shortened protocol, T1mapping, CMR

\section{Background}

Cardiac MRI (CMR) is a very established technique in clinical practice. However, the examination remains time consuming and requires expertise of both the technician and the reader since conventional techniques such as T2 weighted imaging and Late-Gadolinium-Enhancement mainly allow for qualitative or semi-qualitative analysis

\footnotetext{
* Correspondence: j.nadjiri@gmx.net

${ }^{1}$ Department of Diagnostic and Interventional Radiology, Klinikum rechts der Isar, School of Medicine, Technical University of Munich, Ismaninger Str. 22, 81675 Munich, Germany

Full list of author information is available at the end of the article
}

[1-3]. Depending on the given routine in a CMR laboratory, an examination to rule out structural heart disease or myocarditis can take $30 \mathrm{~min}$ up to an hour or more [4]. This limits the number of CMR examinations that can be offered to referring physicians. As a consequence, patients might be scheduled for CMR much later than the onset of symptoms delaying correct diagnosis and possibly reducing sensitivity of the examination due to chronification $[4,5]$. Therefore, short examination times in CMR are mandatory to offer this highly potent diagnostic test to a broad population of patients with clinical indication. This is especially relevant for clinical routine

(C) The Author(s). 2019 Open Access This article is distributed under the terms of the Creative Commons Attribution 4.0 International License (http://creativecommons.org/licenses/by/4.0/), which permits unrestricted use, distribution, and 
use with an inhomogeneous patient population with many subjects having a low pre-test probability for myocardial disease.

Quantitative approaches like mapping sequences are now established in daily routine CMR $[1,3,6-8]$. T1 and T2 mapping allow for a per voxel calculation of the absolute myocardial relaxation time eliminating the need of comparison with assumed healthy tissue. Both techniques show excellent diagnostic performance regarding specific myocardial diseases such as infarction or myocarditis as well as storage diseases. Additionally, these sequences also bear prognostic value [9-11].

Native T1 mapping is able to detect edema, hemorrhage, siderosis, lipid and protein disposition as well as fibrosis [6, 12-14]. This overlap of different myocardial changes being detected by $\mathrm{T} 1$ mapping indicates a potential for utilization as a search sequence $[7,15,16]$.

The purpose of the present investigation was to retrospectively evaluate whether a very brief CMR protocol sufficiently distinguishes patients with relevant myocardial changes with need for further examination from healthy subjects.

\section{Methods}

\section{Study population}

All consecutive patients receiving clinically indicated CMR for left ventricular evaluation between October 2015 and October 2017 were retrospectively evaluated. The study has been approved by the local ethics committee of the Medical Faculty of the Technische Universität München. Patients with missing T1 mapping were excluded as well as patients with evaluation of cardiac masses. All patients in this study received full contrast CMR.

\section{Imaging}

Cardiac imaging was performed using a $3.0 \mathrm{~T}$ Philips Ingenia clinical dual-source RF transmission MR system (Philips Healthcare, Best, the Netherlands) as described before [17]. T1 mapping was performed as described before [17]. As a reference for mapping techniques, scans of healthy volunteers are highly recommended $[7,15,16]$. Mean T1 was 1175 ms measured in 13 healthy volunteers with the same protocol. T2 values in healthy volunteers were not available.

For the T2 mapping Gradient Spin Echo (GraSE) sequence, a train of spin-echoes is generated by several $180^{\circ}$ radiofrequency pulses and each individual spin echo is acquired with an EPI readout. A six-echo variant was used. A dual inversion recovery black blood module was applied to null the signal of the blood. Electrocardiography-gated breath-hold GraSE sequences were acquired as follows: $\mathrm{TR}=1 \mathrm{RR}$ interval, TE $10-100 \mathrm{~ms}$ (9 echoes), flip angle $90^{\circ}$. Acquired voxel size was $2.0 \times 2.0 \times 8.0 \mathrm{~mm} 3$, matrix was $176 \times 175 \mathrm{~mm}^{2}$. Besides T1 Modified Look Locker Inversion Recovery (MOLLI) and T2 GraSE imaging, all patients received a clinical cardiac MR protocol adjusted to the respective clinical issue. Cine sBTFE imaging, T2 TSE dark-blood imaging and Early-GadoliniumEnhancement (EGE) (5 min after Gd application) and Late-Gadolinium-Enhancement (LGE) imaging (3D IR GRE or 2D PSIR) were performed in all subjects according to the institutional standard protocol. Dual source radiofrequency transmission was used for cine imaging; FOV was $320 \times 410 \mathrm{~mm}$, voxel size was $1.9 \times 1.19 \times 8 \mathrm{~mm}$, TR was $2.6 \mathrm{~ms}$ and TE $1.2 \mathrm{~ms}$. Flip angle was $45^{\circ}$ and sense factor was 2 . All images were obtained using retrospective gating. For all sequences the inline function arrhythmia rejection was used.

\section{Image analysis}

LV-function analysis was done with sBTFE cine imaging in short axis view using the Philips Intellispace software (Ver. 8). LV-parameters were documented in the standard clinical CMR report. After review, this data was accepted as a study parameter. Myocardial evaluation was done using the AHA/ACC 17-segment model excluding segment 17 to avoid partial volume effects. Assessment of T2-weighted images, T1- and T2- relaxation time as well as extracellular volume (ECV) was based on corresponding short axes and measured for each of the abovementioned $16 \mathrm{seg}$ ments. For that purpose, regions of interest (ROI) were semi-automatically delineated using the Philips Intellispace software (Ver. 8). For reference in T2w imaging an additional ROI within skeletal muscle $\left(\mathrm{ROI}>30 \mathrm{~mm}^{2}\right)$ was delineated. T2w images were semi-quantitatively assessed by calculation of ratio of signal intensity of myocardium and skeletal muscle as described before [18]. At our institution the local threshold for $\mathrm{T} 2 \mathrm{w}$ ratio was defined with 2.5. Imaging for EGE, LGE and wall movement disorders were evaluated visually and dichotomously in SBTFE cine imaging. For calculation of ECV a synthetic haematocrit was estimated as proposed by Treibel et al. [19]; however, an adjustment using curve fitting was necessary to compensate for $3 \mathrm{~T}$ compared to 1.5 :

Estimated haematocrit $=\left(\frac{973}{\text { Native T1 relaxation time of blood }}\right)-0,1232$.

ECV was calculated as described before [8, 20]. For analysis in every patient the segment with highest T1- and T2 relaxation times as well as ECV und $\mathrm{T} 2$ ratios were defined; all highest segments were then reviewed again. Segments with artefacts were carefully excluded and replaced by the segment with the second highest value without artefact. These variables were defined as T1max, T2max and ECVmax, 
respectively. Selection of the segments with highest values of T1, T2 and ECV allows to compare focal myocardial alterations with global ones. All clinical reports were reviewed by a Level III CMR reader (certified by the European Association of Cardiovascular Imaging).

For the study analysis CMR data was evaluated and ROIs were delineated by a CMR reader in training; this data was later again reviewed by a Level III CMR reader. As binary endpoint we defined no myocardial finding in the CMR report, normal signal characteristics in all standard sequences (T2w DB, EGE and LGE) and - depending on subgroup analysis - also LV-parameters. Pericardial effusion or thickening without myocardial involvement were not regarded as myocardial pathology and therefore categorized as no finding for this analysis. In case of equivocal findings definite decision was made by the Level III CMR reader.

\section{Statistical analysis}

Continuous variables were expressed as mean \pm standard deviations (SD). The tested data has visually been evaluated for normal distribution. Two-sided $t$ test was utilized for exploratory testing for gender differences of native and contrast enhanced $\mathrm{T} 1$ relaxation. For assessment of correlation Pearson's coefficient has been determined; values of $0.3-0.5$ are regarded as low, $0.5-0.7$ as moderate, $0.7-0.9$ as high and values of $0.9-1$ as very high correlation as suggested [21]. Receiver operator characteristic (ROC) analysis was performed using the method of DeLong [22]. As endpoint for ROC-analysis we defined no myocardial finding in the CMR report within the entire study population. In subgroup analyses in patients with no LV-dysfunction the endpoint was any myocardial pathologic finding in the CMR report excluding LVparameters. And in subgroup analyses in patients with LV-dysfunction the endpoint was any further myocardial pathology besides LV-dysfunction. Optimal cut-off values were calculated by maximization of the sum of sensitivity and specificity. As global level of significance a $p$ value of 0.05 was accepted. The statistical package $R$ version 3.0.3 [23] was used for statistical analysis.

\section{Results}

\section{Study population}

In total 160 patients were included in the study. $66 \%$ of the patients were male. Mean age at examination was $45 \pm 16$ years. In Table 1 a breakdown of CMR indications is provided. In Figs. 1 and 2 image examples of a severe case of myocarditis with follow-up is provided.
Table 1 Lists general patients' information with a break down of the Cardiac MRI (CMR) indication written on requesting form Patients information $(n=160)$

\begin{tabular}{ll}
\hline General information & \\
Age & $45 \pm 16$ years \\
Male gender & $106(66 \%)$ \\
Synthetic haematocrit & $0.41 \pm 0.028$ \\
Indication for CMR & \\
Acute myocarditis & $81(51 \%)$ \\
Chronic myocarditis & $13(8 \%)$ \\
Structural heart disease & $9(6 \%)$ \\
Unspecific clinical indications (e.g. check-up) & $21(13 \%)$ \\
Systemic disease & $19(12 \%)$ \\
Ischemic heart disease & $17(11 \%)$ \\
\hline
\end{tabular}

\section{CMR findings}

In $69 \%(n=111)$ of patients there was a positive finding in CMR. 51\% $(n=82)$ had either focal or global left ventricular (LV) dysfunction. In $34 \% \quad(n=55)$ increased myocardial T2w signal was found. In 43 patients $(n=27 \%)$ EGE was present. In $40 \%(n=65)$ of patients positive LGE was found. In Table 2 a breakdown of CMR diagnosis as documented in the final CMR report is provided. Table 2 shows further findings from CMR in more detail. All diagnoses were made with final consensus and agreement of the department of Cardiology at daily conferences.

\section{Results of ROC analyses - comparison of the sequences ROC analysis in the entire study population $(n=160)$}

Area under the curve (AUC) for native T1 mapping evaluating the segment with highest $\mathrm{T} 1$ relaxation time (T1max) was $78 \%$, for ECV (ECVmax) 73\%; $p<$ $0.001,95 \%$ CI $[71-85 \%$ and $65-81 \%]$. Optimal cutoff was $1335 \mathrm{~ms}$ for native T1 mapping with a sensitivity of $67 \%$ and a specificity of $67 \%$. Best cut-off for ECV was $34 \%$ with a sensitivity of $67 \%$ and a specificity of $67 \%$. Sensitivity for the combined use of T1 mapping and sBTFE cine imaging was $97 \%$. Additional T2 mapping was available for $110 \mathrm{pa}$ tients: ROC was 69\%; $p<0.001,95 \%$ CI [59-78\%]. Sensitivity was $72 \%$ and specificity was $60 \%$ with optimal cut-off of $55 \mathrm{~ms}$.

\section{ROC analysis in patients with $(n=82)$ and without $(n=78)$ LV dysfunction}

In patients with LV dysfunction AUC was $74 \%$ for T1max and $73 \%$ for ECV; $p<0.001$ for both, 95\% CI [61-87\% and 59-88\%]. Optimal cut-off for T1max was $1346 \mathrm{~ms}$ with a sensitivity of $60 \%$ and specificity of $63 \%$. For ECVmax in patients with LV dysfunction optimal 

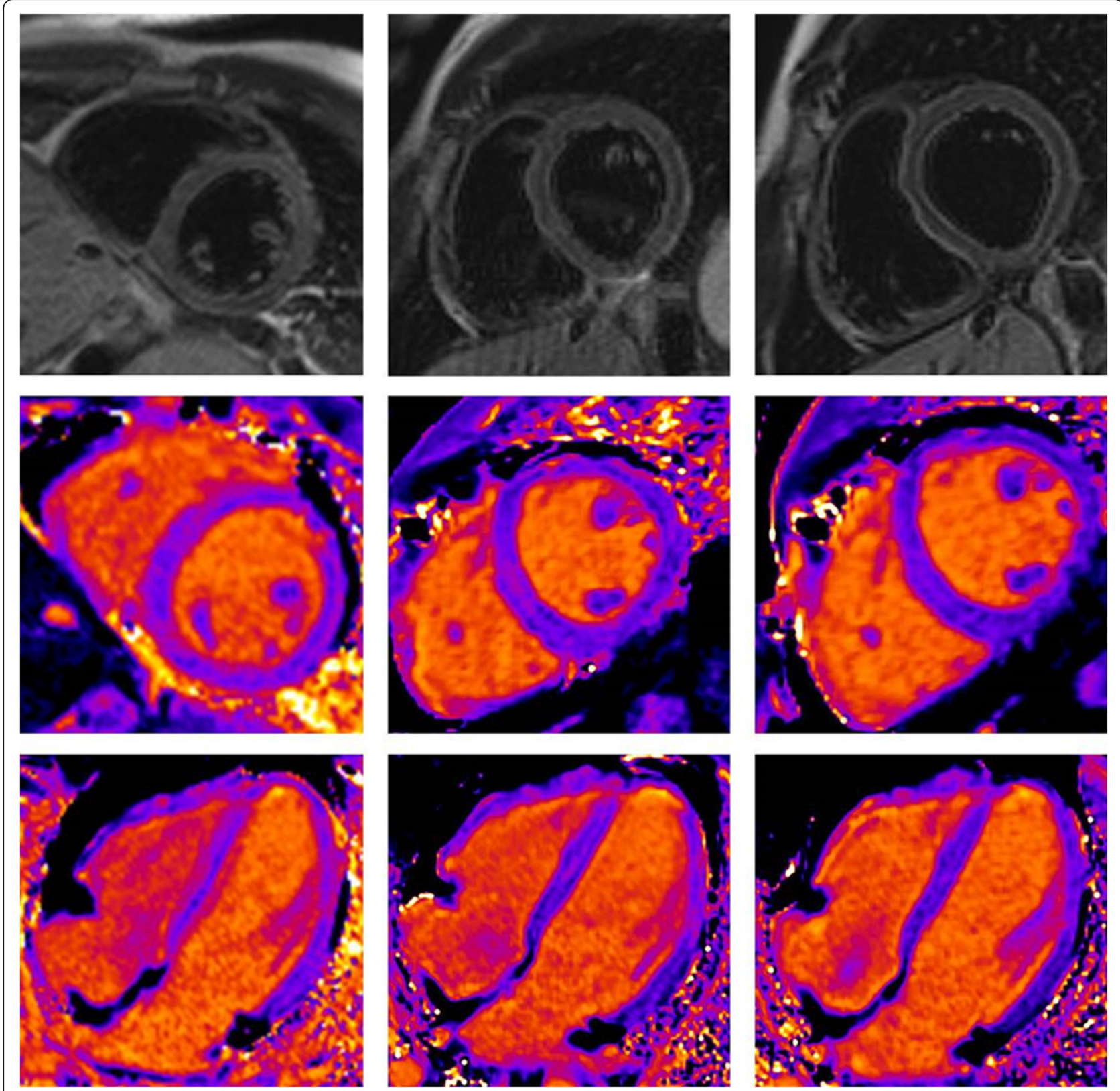

Fig. 1 Example of an 18-year-old male patient with severe myocarditis. Native sequences: T2w dark blood (top row) and native T1 mapping in short axis (middle row) and 4 chamber view (lower row) at acute state, 8 and 16 weeks follow up exam (columns from left to right). Highest T1 relaxation time in this patient was 4 standard deviations above values of volunteers and well above our threshold in acute state and within normal range at follow-up. It is of note that T2w imaging appears to be normal due to inflammatory involvement of the whole heart. Even the ratio of myocardium and skeleton muscle signal intensity was normal as a result of accompanying myositis

cut-off was $34 \%$ with a sensitivity and specificity of 70 and $69 \%$ respectively.

In patients without LV dysfunction AUC for T1max was 82 and 75\% for ECVmax; $p<0.001$ for both. For difference of the curves $\mathrm{p}$ was 0.25 . Optimal cut-off for T1max was $1336 \mathrm{~ms}$ and $34 \%$ for ECV. Sensitivity and specificity for T1max was 74 and $74 \%$. For ECVmax sensitivity and specificity was 67 and $68 \%$.

\section{Comparison in patients with no $L V$ dysfunction and additional T2 mapping $(n=56)$}

AUC was $82 \%$ for native T1 mapping evaluating the segment with highest relaxation times; $p<0.001,95 \%$ CI [7194\%]. Optimal cut-off was $1338 \mathrm{~ms}$ with a sensitivity of $77 \%$ and a specificity of $74 \%$.

For calculated ECV, AUC was $79 \%$ for the segment with highest value; $p<0.001,95 \%$ CI [66-92\%]. Optimal 

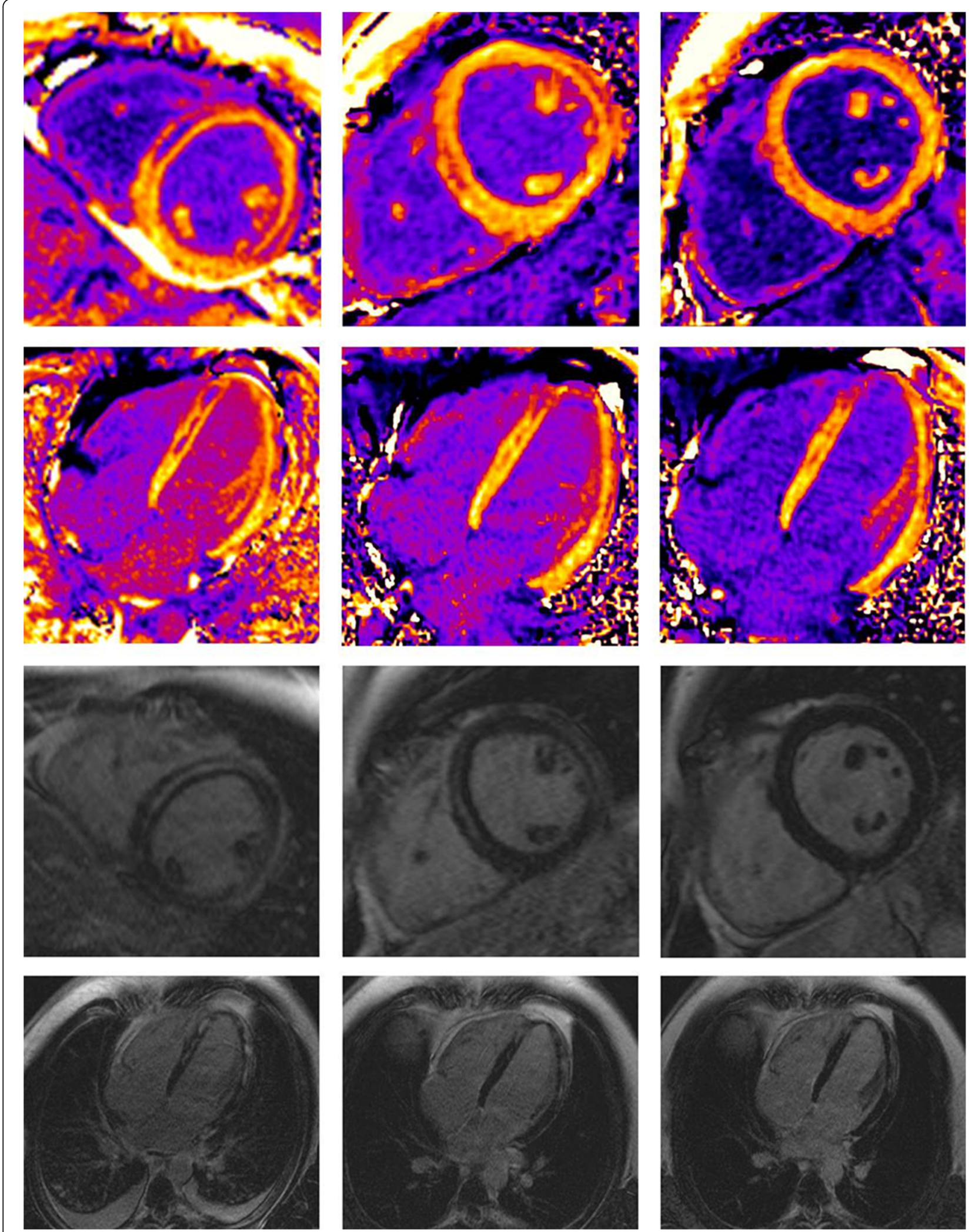

Fig. 2 Example of an 18-year-old male patient with severe myocarditis. Contrast enhanced sequences: CE T1 mapping in short axis and 4 chamber view (first two rows) and Late-Gadolinium-Enhancement (last two rows) at acute state and 8 and 16 weeks follow up exam (columns from left to right). Maximum ECV was $51 \%$ in acute state in the anterior septal wall and $25 \%$ at 16 week follow up 
Table 2 Lists general patients' CMR information together with a breakdown of the diagnoses reported by cardiac MRI (CMR)

\begin{tabular}{|c|c|}
\hline \multicolumn{2}{|l|}{ CMR data $(n=160)$} \\
\hline \multicolumn{2}{|l|}{ LV parameters } \\
\hline Endsystolic volume & $74 \pm 53 \mathrm{~m}$ \\
\hline Enddiastolic volume & $\begin{array}{l}158 \pm 60 \\
\mathrm{ml}\end{array}$ \\
\hline Ejection fraction & $55 \pm 12 \%$ \\
\hline Regional wall motion abnormality & $36(23 \%)$ \\
\hline \multicolumn{2}{|l|}{ Myocardial characterisation } \\
\hline Mean T1 relaxation time ${ }^{a}$ & $\begin{array}{l}1266 \pm 89 \\
\mathrm{~ms}\end{array}$ \\
\hline Maximum $\mathrm{T} 1$ relaxation time ${ }^{\mathrm{b}}$ & $\begin{array}{l}1343 \pm 98 \\
\mathrm{~ms}\end{array}$ \\
\hline $\begin{array}{l}\text { Number of segments with increased T1 relaxation } \\
\text { time(> } 1300 \text { ms[first quartile of the study population]) }\end{array}$ & $2.5 \pm 4.1$ \\
\hline Mean T2 relaxation time ${ }^{a}$ & $\begin{array}{l}49.7 \pm 5.4 \\
\mathrm{~ms}\end{array}$ \\
\hline Maximum $\mathrm{T} 2$ relaxation time ${ }^{\mathrm{b}}$ & $\begin{array}{l}58.1 \pm 9.7 \\
\mathrm{~ms}\end{array}$ \\
\hline $\begin{array}{l}\text { Number of segments with increased } \mathrm{T} 2 \text { relaxation time } \\
\text { (>55 ms) }\end{array}$ & $2.3 \pm 3.9$ \\
\hline Mean ECV ${ }^{a}$ & $\begin{array}{l}30.6 \pm \\
4.6 \%\end{array}$ \\
\hline Maximum ECV ${ }^{b}$ & $\begin{array}{l}34.3 \pm 5.5 \\
\mathrm{~ms}\end{array}$ \\
\hline $\begin{array}{l}\text { Number of segments with increased ECV relaxation time } \\
(>34 \%)\end{array}$ & $4.3 \pm 5.4$ \\
\hline $\begin{array}{l}\text { Number of segments with increased signal intensity in } \\
T 2 w D B\end{array}$ & $1.8 \pm 2.7$ \\
\hline Positive EGE & $43(27 \%)$ \\
\hline Positive LGE & 65 (41\%) \\
\hline \multicolumn{2}{|l|}{ Diagnosis from CMR report } \\
\hline Acute myocarditis & $19(12 \%)$ \\
\hline Chronic myocarditis & $21(13 \%)$ \\
\hline Structural heart disease & $18(11 \%)$ \\
\hline Unclear or unspecific diagnosis & $26(16 \%)$ \\
\hline Systemic disease & $15(9 \%)$ \\
\hline Ischemic heart disease & $12(8 \%)$ \\
\hline $\begin{array}{l}\text { No finding in CMR report or minor unspecific findings } \\
\text { (e.g. slightly increased amount of pericardial fluid) }\end{array}$ & 49 (31\%) \\
\hline
\end{tabular}

${ }^{a}$ average of segment $1-16,{ }^{b}$ segment with highest value

cut-off was $34 \%$ with a sensitivity of $69 \%$ and a specificity of $70 \%$.

AUC was 60\% for T2 mapping (T2max) for the segment with maximum relaxation time, $p=0.1,95 \% \mathrm{CI}$ [45-76\%]. Optimal cut-off was $55 \mathrm{~ms}$ with a sensitivity of $54 \%$ and a specificity of $60 \%$.

Difference of the AUC was $22 \%$ in favour of $\mathrm{T} 1 \mathrm{max}$ over T2max; $p=0.012$. For T1max the difference of the AUC was $3 \%$ in comparison to ECVmax; $p=0.7$. Difference of AUC between T2max and ECVmax was
$19 \%$ in favour of ECVmax; $p=0.013$. ROCs for the three quantitative sequences are provided in Fig. 3.

\section{Analysis of individual benefit from full contrast CMR}

In our study $51 \%(n=82)$ patients had LV dysfunction and therefore requiring full contrast enhanced CMR. $49 \%(n=78)$ of the patients showed no LV dysfunction. In this cohort $40 \%(n=31)$ had a T1max of less than $1300 \mathrm{~ms}$ (first quartile of the study population) and thus below the optimal cut-off. In this group 90\% $(n=28)$ had no pathological finding in their final report and therefore did not benefit from the full contrast CMR protocol. In the group of patients with a T1max below $1300 \mathrm{~ms}, 10 \%(n=3)$ had a pathologic finding; all three cases were consistent with chronic myocarditis. From the cohort with T1max higher than $1300 \mathrm{~ms} 45 \%(n=21)$ had no findings in the CMR report, thus the full CMR exam did not provide any additional information. However, from the same cohort with T1max higher than $1300 \mathrm{~ms} 55 \%$ had a pathologic finding and therefore benefitted from full contrast CMR. In our study population $70 \%$ of the patients $(n=111)$ had a finding in their final CMR report and 30\% $(n=49)$ did not. Of these 49 patients $57 \% \quad(n=28)$ could be identified as patients who would not benefit from full contrast CMR by T1 mapping and cine imaging alone. In patients without LV dysfunction the negative predictive value of native T1 mapping was 90\%. A detailed illustration of the examination outcome is provided in Fig. 4.

\section{Discussion}

The purpose of the present investigation was to evaluate a shortened CMR protocol in clinical routine practice.

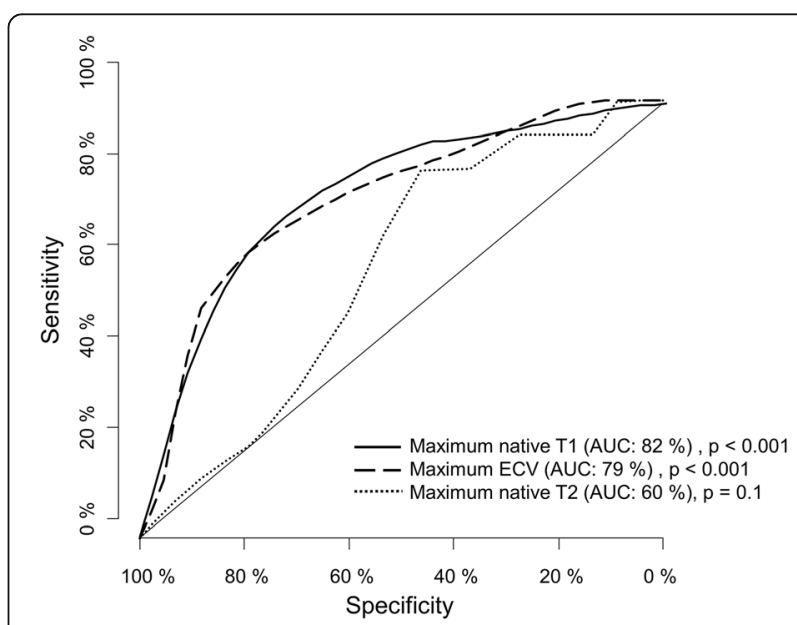

Fig. 3 shows a ROC analysis of the three quantitative techniques ( $T$ 1 mapping [solid line], T2 mapping [dotted line] and extracellular volume [dashed line]) in patients with no left ventricular dysfunction where additional T2 mapping was available $(n=56)$ 


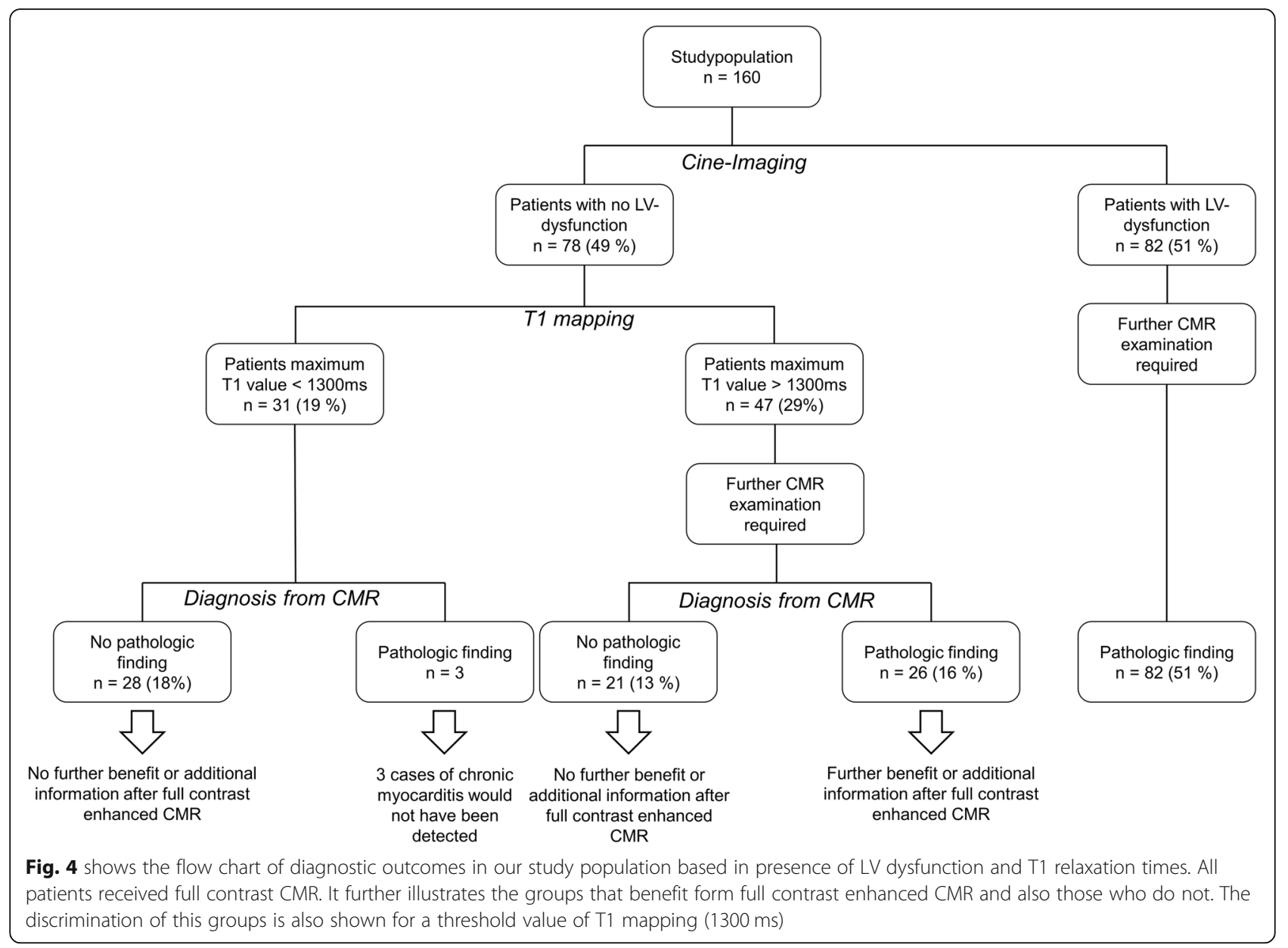

Therefore, a very heterogenous patient collective was investigated. The main findings of this study are i) T1 mapping is superior to T2 mapping in detection of unspecific myocardial pathology in a heterogenous study population of patients without LV-dysfunction and ii) a shortened protocol comprising only $\mathrm{T} 1$ mapping and LV-function analysis discriminates patients who will benefit from a full contrast enhanced CMR protocol from those who do not with sufficient confidence.

\section{Need for shorter examinations}

In clinical practice CMR, amongst a few other applications, is considered to be the most time consuming and therefore economically challenging examination. Due to lengthy protocols, emergency examinations cannot easily be integrated into a busy schedule even if there are drop outs. Further, the examination is technically challenging and labor intensive. In an optimal setting a CMR examination occupies one trained physician and up to two technicians $[4,24]$. This further stresses an effective and economic use of MRI scanners. Long examination times also hamper patient comfort. As a consequence, important CMR examinations can be delayed in the clinical routine while CMR reports are often urgently needed for clinical decision making [5]. Therefore, shorter CMR protocols are desirable allowing more patients to benefit from the high diagnostic value that makes CMR the diagnostic gold standard in detection of several cardiovascular diseases. Yet, data on rigorously shortened CMR protocols is very rare.

\section{Evaluation of the study results}

The suggested protocol in this study comprises a quantitative approach for myocardial assessment and a combination of quantitative and qualitative evaluation of the left ventricle. In our study population T1 mapping was superior to T2 mapping and was therefore further investigated in the shortened CMR protocol. Prolongation of T1 relaxation time can be found in several entities of myocardial pathology such as edema, fibrosis, protein deposition and others [7]. In contrast, T2 relaxation times are mainly increased in presence of edema and slightly in case of fibrosis. Additionally, a broad range of myocardial T2 values can be found in healthy volunteers complicating to set a strong threshold value $[25,26]$. It is to assume that those are the two main reasons why 
T1 mapping was superior to T2 mapping in detection of patients with a pathological finding in the final CMR report in our heterogenous study population. Further, we also found good diagnostic performance of ECV evaluation. However, determination of ECV requires T1 mapping measurement before i.v. gadolinium application and approximately $10 \mathrm{~min}$ after. For that reason, this technique does not necessarily lead to a shortened examination time. Yet, native T1 mapping was not inferior to ECV measurement in our population; this is of note since the latter is a contrast enhanced technique with partially inherent information about EGE/LGE [27]. Additionally, non-enhanced protocols are desirable due to the growing knowledge on deposition of MR contrast agents in the human body [28, 29] and increasing concerns against $\mathrm{Gd}$ application on the patients' side.

One approach to shorten CMR examination time is to predict at an early state which patient will benefit from the full contrast enhanced examination and which will not. In our collective every third patient did not benefit from a full contrast enhanced CMR. 57\% of those subjects were correctly identified by native $\mathrm{T} 1$ mapping and cine imaging alone. Regarding the entire study population, the shortened protocol correctly ruled out pathology in every fifth patient.

Yet, three cases of pathologic findings were overlooked using the shortened sequence protocol. Those three cases were consistent with chronic myocarditis. It is of note that this diagnosis does not represent acute myocardial damage with inherent treatment indication. Therefore, clinical relevance is questionable if there is no other pathologic myocardial alteration present like LV dysfunction or others. Additional T2 mapping would not have detected these 3 cases of chronic myocarditis. In summary, no case of acute myocardial damage with inherent obligation to treat has been missed.

\section{Future applications of the shortened protocol - can it be used as a screening method?}

Even though the proposed shortened CMR protocol yielded satisfying results, a standardized use in clinical routine is somewhat challenging. Application as a screening protocol with image analysis after the scan would neither be cost nor time effective since approximately $70 \%$ of the patients would have to be rescanned with the need for full contrast CMR. Although a partial scan has already been acquired and those sequences could be omitted in the full contrast scan, positioning of the patient in the scanner and geometrical planning of the sequences is a very time-consuming part of the examination. As a result, net scanner occupancy would not be reduced.

Another approach would be to immediately evaluate the image data including determination of myocardial
T1 values as well as LV-parameters while the patient is still inside the scanner. If both $\mathrm{T} 1$ relaxation time and LV functional parameters are within normal range, further sequences and contrast application can be omitted with fair confidence. According to our mixed cohort, this will assumably be the case in approximately every fifth patient. The saved scanning time might be used to increase the number of CMR examinations offered to referring physicians.

The disadvantage is a reduced predictability of the scan length. Further, this also requires a physician permanently present at the scanner to be effective.

Assuming that a full protocol takes $60 \mathrm{~min}$ and the proposed shortened protocol $30 \mathrm{~min}$, the second scenario would allow to stop after the shortened protocol in 31 cases from our study population. This would safe $15.5 \mathrm{~h}(10 \%)$ of scanner time at the cost of $2 \%$ incorrect diagnoses only comprising chronic myocarditis of which clinical relevance and evidence-based treatment consequence, respectively, remain unclear.

Additionally, the presented findings might be of importance when shortened protocols are a necessity instead of a choice, for example when the patients' clinical condition does not allow for a time consuming full CMR protocol or if image quality of contrast enhanced sequences is hampered due to technical issues. Our study results indicate that in these cases the radiologist can rely on cine and mapping imaging for making a diagnosis.

Besides the economic relevance of the findings of this study, the diagnostic potential of the proposed shortened protocol has to be further evaluated prospectively in a larger multicentric study population.

It is of note that in this study sBTFE cine imaging was used for LV evaluation. Real-time imaging could further strongly shorten examination time. Recent studies have shown that measured values showed high accuracy and reliability [30]. Therefore, a protocol comprising only T1 mapping and real-time cine imaging seems very promising since examination time can probably be reduced to less than $15 \mathrm{~min}$.

\section{Limitations}

This is a retrospective single centre study. The study results are limited due to the relatively small size of the study population. Even though, the purpose of the study was to evaluate the shortened protocol for the left ventricle in daily practice conditions and therefore requiring a heterogenous cohort the composition of the study population might be dependent of statistic variations as might the subsequent results. Further, no validation against solid markers was available (e.g. laboratory values such as troponin or tissue from myocardial biopsy). In the study population right ventricular pathology was excluded. The shortened protocol would assess RV-wall 
movement disorders but the spatial resolution of the proposed T1 mapping sequence is probably too low to allow full and correct diagnosis on the RV limiting the method to LV evaluation.

\section{Conclusions}

A shortened CMR protocol comprising only T1 mapping and LV function analysis seems to sufficiently rule out myocardial alterations in our study. Sensitivity was $98 \%$; only three cases of chronic myocarditis with normal LVfunction were overlooked, yet therapeutic consequences of this entity remain uncertain. The proposed protocol might enhance cost-effectiveness by shorter examination time and by omitting contrast application without missing relevant findings for certain clinical questions, especially when pre-test probability is low. Shorter examination times optimize patients' comfort.

\section{Abbreviations}

AUC: Area Under the Curve; CMR: Cardiac Magnetic Resonance imaging; DB: Dark Blood; ECV: Extra Cellular Volume; ECVmax: Highest Extra Cellular Volume; EGE: Early-Gadolinium-Enhancement; EPI: Echo-planar imaging; GraSE : Gradient Spin Echo; GRE: Gradient Echo; IR: Inversion Recovery; LGE: Late-Gadolinium-Enhancement (LGE); LV: Left Ventricle; MOLLI: Modified Look Locker Inversion Recovery; ms: milliseconds; PSIR: Phase Sensitive Inversion Recovery; ROC: Receiver Operator Characteristic; ROI: Regions Of Interest; sBTFE: Sense Balanced Turbo Field Echo; SD: Standard Deviation; T1 max: Highest $\mathrm{T} 1$ relaxation time in $\mathrm{ms} ; \mathrm{T} 2 \mathrm{max}$ : Highest $\mathrm{T} 2$ relaxation time in ms; TE: Echo Time; TR: Repetition Time; TSE: Turbo Spin Echo

\section{Acknowledgments}

We are indebted to the medical and technical staff members of the magnetic resonance laboratory for their invaluable contribution.

\section{Authors' contributions \\ $J N, A Z, A S, A S 2, M E, F W$ and DP contributed in designing the study, analyzation of results, interpretation of results drafting and revising the manuscript. MR, KL and EJR contributed in providing the resources, designing the study, analyzation of results, drafting and revising the manuscript. All authors have read and approved the manuscript.}

\section{Funding}

No Funding.

\section{Availability of data and materials}

The datasets used and/or analysed during the current study are available from the corresponding author on reasonable request.

\section{Ethics approval and consent to participate}

All procedures performed in studies involving human participants were in accordance with the ethical standards of the institutional and national research committee and with the 1964 Helsinki declaration and its later amendments or comparable ethical standards.

The study design was approved by the local ethics committee (Ethikkommission der Medizinischen Fakultät der Technischen Universität München, School of Medicine)

This is a retrospective study; all patients underwent clinically indicated MR examination. Informed consent was waived by the local ethics committee.

\section{Consent for publication}

This is a retrospective study; all patients underwent clinically indicated MR examination. All personal details were anonymized for evaluation and publication. Informed consent was waived by the local ethics committee.

\section{Competing interests}

The authors have nothing to disclose. This is an investigator-driven study; there is no involvement from outside the departments.

\section{Author details}

${ }^{1}$ Department of Diagnostic and Interventional Radiology, Klinikum rechts der Isar, School of Medicine, Technical University of Munich, Ismaninger Str. 22, 81675 Munich, Germany. '2Department of Cardiology, Klinikum rechts der Isar, School of Medicine, Technical University of Munich, Ismaninger Str. 22, 81675 Munich, Germany.

Received: 18 April 2019 Accepted: 9 July 2019

Published online: 24 July 2019

\section{References}

1. Ferreira VM, Piechnik SK, Dall'Armellina E, Karamitsos TD, Francis JM, Ntusi N, Holloway C, Choudhury RP, Kardos A, Robson MD, et al. T(1) mapping for the diagnosis of acute myocarditis using CMR: comparison to T2-weighted and late gadolinium enhanced imaging. JACC Cardiovasc Imaging. 2013;6(10):1048-58.

2. Friedrich MG, Sechtem U, Schulz-Menger J, Holmvang G, Alakija P, Cooper LT, White JA, Abdel-Aty H, Gutberlet M, Prasad S, et al. Cardiovascular magnetic resonance in myocarditis: a JACC White paper. J Am Coll Cardiol. 2009:53(17):1475-87.

3. Nassenstein K, Nensa F, Schlosser T, Bruder O, Umutlu L, Lauenstein T, Maderwald S, Ladd ME. Cardiac MRI: T2-mapping versus T2-weighted dark-blood TSE imaging for myocardial edema visualization in acute myocardial infarction. Rofo. 2014;186(2):166-72.

4. Kramer CM, Barkhausen J, Flamm SD, Kim RJ, Nagel E, Society for Cardiovascular Magnetic Resonance Board of Trustees Task Force on Standardized P. Standardized cardiovascular magnetic resonance imaging (CMR) protocols, society for cardiovascular magnetic resonance: board of trustees task force on standardized protocols. J Cardiovasc Magn Reson. 2008;10:35.

5. Laissy J-P, Hyafil F, Feldman L, Juliard J-M, Schouman-Claeys E, Steg PG, Faraggi M. Differentiating acute myocardial infarction from myocarditis: diagnostic value of early-and delayed-perfusion cardiac MR imaging. Radiology. 2005;237(1):75-82.

6. Ferreira VM, Piechnik SK, Dall'Armellina E, Karamitsos TD, Francis JM, Choudhury RP, Friedrich MG, Robson MD, Neubauer S. Non-contrast T1mapping detects acute myocardial edema with high diagnostic accuracy: a comparison to T2-weighted cardiovascular magnetic resonance. J Cardiovasc Magn Reson. 2012;14:42.

7. Moon JC, Messroghli DR, Kellman P, Piechnik SK, Robson MD, Ugander M, Gatehouse PD, Arai AE, Friedrich MG, Neubauer S, et al. Myocardial T1 mapping and extracellular volume quantification: a Society for Cardiovascular Magnetic Resonance (SCMR) and CMR working Group of the European Society of cardiology consensus statement. J Cardiovasc Magn Reson. 2013;15:92.

8. Nadjiri J, Nieberler H, Hendrich E, Greiser A, Will A, Martinoff S, Hadamitzky M. Performance of native and contrast-enhanced T1 mapping to detect myocardial damage in patients with suspected myocarditis: a head-to-head comparison of different cardiovascular magnetic resonance techniques. Int J Cardiovasc Imaging. 2017;33(4):539-47.

9. Fontana M, White SK, Banypersad SM, Sado DM, Maestrini V, Flett AS, Piechnik SK, Neubauer S, Roberts N, Moon JC. Comparison of T1 mapping techniques for ECV quantification. Histological validation and reproducibility of ShMOLLI versus multibreath-hold T1 quantification equilibrium contrast CMR. J Cardiovasc Magn Reson. 2012;14(1):88.

10. Langhans B, Nadjiri J, Jahnichen C, Kastrati A, Martinoff S, Hadamitzky M. Reproducibility of area at risk assessment in acute myocardial infarction by T1- and T2-mapping sequences in cardiac magnetic resonance imaging in comparison to Tc99m-sestamibi SPECT. Int J Cardiovasc Imaging. 2014;30(7):1357-63.

11. Nadjiri J, Nieberler H, Hendrich E, Will A, Pellegrini C, Husser O, Hengstenberg C, Greiser A, Martinoff S, Hadamitzky M. Prognostic value of T1-mapping in TAVR patients: extra-cellular volume as a possible predictor for peri- and post-TAVR adverse events. Int J Cardiovasc Imaging. 2016;32(11):1625-33.

12. Karamitsos TD, Piechnik SK, Banypersad SM, Fontana M, Ntusi NB, Ferreira VM, Whelan CJ, Myerson SG, Robson MD, Hawkins PN, et al. Noncontrast T1 
mapping for the diagnosis of cardiac amyloidosis. JACC Cardiovasc Imaging. 2013:6(4):488-97.

13. Pedersen SF, Thrysoe SA, Robich MP, Paaske WP, Ringgaard S, Botker HE, Hansen ES, Kim WY. Assessment of intramyocardial hemorrhage by T1-weighted cardiovascular magnetic resonance in reperfused acute myocardial infarction. J Cardiovasc Magn Reson. 2012;14(1):59.

14. Ruberg FL. T1 mapping in cardiac amyloidosis: can we get there from here? JACC Cardiovasc Imaging. 2013;6(4):498-500.

15. Messroghli DR, Moon JC, Ferreira VM, Grosse-Wortmann L, He T, Kellman P, Mascherbauer J, Nezafat R, Salerno M, Schelbert EB, et al. Correction to: clinical recommendations for cardiovascular magnetic resonance mapping of $\mathrm{T} 1, \mathrm{~T} 2, \mathrm{~T}_{2}^{*}$ and extracellular volume: a consensus statement by the Society for Cardiovascular Magnetic Resonance (SCMR) endorsed by the European Association for Cardiovascular Imaging (EACVI). J Cardiovasc Magn Reson. 2018;20(1):9.

16. Messroghli DR, Moon JC, Ferreira VM, Grosse-Wortmann L, He T, Kellman P, Mascherbauer J, Nezafat R, Salerno M, Schelbert EB, et al. Clinical recommendations for cardiovascular magnetic resonance mapping of $\mathrm{T} 1, \mathrm{~T} 2, \mathrm{~T}^{*}$ and extracellular volume: a consensus statement by the Society for Cardiovascular Magnetic Resonance (SCMR) endorsed by the European Association for Cardiovascular Imaging (EACVI). J Cardiovasc Magn Reson. 2017;19(1):75.

17. Rasper M, Nadjiri J, Strater AS, Settles M, Laugwitz KL, Rummeny EJ, Huber AM. Cardiac MOLLI T1 mapping at 3.0 T: comparison of patientadaptive dual-source RF and conventional RF transmission. Int J Cardiovasc Imaging. 2017;33(6):889-97.

18. Abdel-Aty H, Boye $P$, Zagrosek A, Wassmuth R, Kumar A, Messroghli D, Bock P, Dietz R, Friedrich MG, Schulz-Menger J. Diagnostic performance of cardiovascular magnetic resonance in patients with suspected acute myocarditis: comparison of different approaches. J Am Coll Cardiol. 2005;45(11):1815-22

19. Treibel TA, Fontana M, Maestrini V, Castelletti S, Rosmini S, Simpson J, Nasis A, Bhuva AN, Bulluck H, Abdel-Gadir A. Automatic measurement of the myocardial interstitium: synthetic extracellular volume quantification without hematocrit sampling. JACC CardiovasC Imaging. 2016;9(1):54-63.

20. Ugander M, Oki AJ, Hsu LY, Kellman P, Greiser A, Aletras AH, Sibley CT, Chen MY, Bandettini WP, Arai AE. Extracellular volume imaging by magnetic resonance imaging provides insights into overt and sub-clinical myocardial pathology. Eur Heart J. 2012;33(10):1268-78.

21. Mukaka M. A guide to appropriate use of correlation coefficient in medical research. Malawi Med J. 2012;24(3):69-71.

22. DeLong ER, DeLong DM, Clarke-Pearson DL. Comparing the areas under two or more correlated receiver operating characteristic curves: a nonparametric approach. Biometrics. 1988;44(3):837-45.

23. Team RDC. R: a language and environment for statistical computing: $R$ Foundation for Statistical Computing; 2010.

24. Plein S, Schulz-Menger J, Almeida A, Mahrholdt H, Rademakers F, Pennell D, Nagel E, Schwitter J, Lombardi M. Training and accreditation in cardiovascular magnetic resonance in Europe: a position statement of the working group on cardiovascular magnetic resonance of the European Society of Cardiology. Eur Heart J. 2011;32(7):793-8.

25. Spieker M, Haberkorn S, Gastl M, Behm P, Katsianos S, Horn P, Jacoby C, Schnackenburg B, Reinecke P, Kelm M. Abnormal T2 mapping cardiovascular magnetic resonance correlates with adverse clinical outcome in patients with suspected acute myocarditis. J Cardiovasc Magn Reson. 2017;19(1):38.

26. von Knobelsdorff-Brenkenhoff $F$, Prothmann $M$, Dieringer MA Wassmuth R, Rudolph A, Utz W, Traber J, Greiser A, Niendorf T, Schulz-Menger J. Current T 1 and T 2 mapping techniques applied with simple thresholds cannot discriminate acute from chronic myocadial infarction on an individual patient basis: a pilot study. BMC Med Imaging. 2016;16(1):35.

27. Abdula G, Sörensson P, Lundin M, Svedin J, Klein M, Kellman P, Sigfridsson A, Ugander M. Synthetic phase sensitive inversion recovery late gadolinium enhancement from post-contrast T1-mapping shows excellent agreement with conventional PSIR-LGE for diagnosing myocardial scar. J Cardiovasc Magn Reson. 2014;16(1):P213.

28. McDonald RJ, McDonald JS, Kallmes DF, Jentoft ME, Murray DL, Thielen KR, Williamson EE, Eckel LJ. Intracranial gadolinium deposition after contrast-enhanced MR imaging. Radiology. 2015;275(3):772-82.
29. Radbruch A. Gadolinium deposition in the brain: we need to differentiate between chelated and Dechelated gadolinium. Radiology. 2018;288(2):434-5.

30. Kido T, Kido T, Nakamura M, Watanabe K, Schmidt M, Forman C, Mochizuk T. Compressed sensing real-time cine cardiovascular magnetic resonance: accurate assessment of left ventricular function in a single-breath-hold. J Cardiovasc Magn Reson. 2016;18(1):50.

\section{Publisher's Note}

Springer Nature remains neutral with regard to jurisdictional claims in published maps and institutional affiliations.
Ready to submit your research? Choose BMC and benefit from:

- fast, convenient online submission

- thorough peer review by experienced researchers in your field

- rapid publication on acceptance

- support for research data, including large and complex data types

- gold Open Access which fosters wider collaboration and increased citations

- maximum visibility for your research: over $100 \mathrm{M}$ website views per year

At $\mathrm{BMC}$, research is always in progress.

Learn more biomedcentral.com/submissions 